\title{
Thermophoretic Deposition of Particles in Gas Flowing over Cold Surfaces
}

\author{
G. K. BATCHELOR* AND C. SHEN $\dagger$ \\ *Department of Applied Mathematics and Theoretical Physics, University of Cambridge, Cambridge, England \\ tInstitute of Mechanics, Chinese Academy of Sciences, Beijing, China
}

Received August 20, 1984; accepted January 2, 1985

\begin{abstract}
The rate at which particles carried in a flowing gas are deposited on a cold surface by thermophoretic movement is studied theoretically. Some exact deductions from the coupled equations for the temperature $(T)$ and particle concentration $(C)$ in the moving fluid show that, in cases in which the particles come from a region in which $T$ and $C$ are uniform with values $T_{0}$ and $C_{0}$, there are strong constraints on the possible values of $C / C_{0}$ at a point at which $T / T_{0}$ is known. This suggests the hypotheses that $C / C_{0}$ is approximately uniform (with value $C_{1} / C_{0}$ say) over a cold isothermal boundary (at temperature $T_{1}$ ), in which event the total particle flux to the boundary is proportional to the total heat flux to the boundary, and that the relation between $C_{1} / C_{0}$ and $T_{1} / T_{0}$ is approximately the same for all flow systems. These hypotheses are tested against the available numerical results for a number of steady flow systems, four of them relating to cold bodies in a uniform stream of gas and two relating to flow in a circular tube downstream from a sudden drop in wall temperature, and are found to represent the exact results with adequate accuracy except when both $T_{1} / T_{0}$ and the thermophoretic coefficient are small. We thus have a means of estimating the total rate of deposition on a cold isothermal boundary from a knowledge of the total heat flux to the boundary in cases in which computation of the distributions of temperature and particle concentration in the fluid is not feasible, although further tests of the hypotheses are desirable. (c) 1985 Academic Press, Inc.
\end{abstract}

\section{INTRODUCTION}

It has been established (see, for example, Kennard (1), Hidy and Brock (2), Friedlander (3)) that the discrete molecular structure of a gas causes small suspended particles to migrate down an ambient temperature gradient with a velocity which is proportional to the temperature gradient. This phenomenon of thermophoresis of aerosol particles is common in nature and in industry. Thermal precipitators are particularly effective in removing small particles with a diameter of a micron or less from a gas stream. Thermophoresis also causes the staining of surfaces of heat exchangers and dirt patterns on the ceilings of kitchens and dining rooms.

There have been relatively few published papers in which the rate of thermophoretic deposition of particles on a solid surface in flow systems of practical interest have been calculated. The authors of these previous papers have considered particular flow sys- tems, and have used numerical methods of calculation. In this paper we shall seek information concerning the rate of thermophoretic deposition which is as general as possible in its application to different flow fields.

The Brownian diffusivity of an aerosol particle $(D)$ is very small, much smaller than any of the gas diffusivities (for mass, momentum, heat), owing to the massive size of a particle by comparison with a gas molecule. For example, for spherical particles of diameter $0.05-1.0 \mu \mathrm{m}$ in air, $D$ ranges from $2.4 \times 10^{-3}$ to $2.8 \times 10^{-5} \mathrm{~m}^{2} / \mathrm{s}$ and the Schmidt number $\nu / D$ from $6 \times 10^{3}$ to 5 $\times 10^{5}$. Effects of particle diffusion are consequently negligible normally, and the movement of a particle is affected by convection with the fluid and thermophoresis only. This results in a strong connection between the distributions of particle concentration and of temperature in the fluid, and leads to the possibility of use of the considerable body of existing analytical, numerical, and experi- 
mental information about the temperature distribution in a variety of flow systems for the purpose of prediction of rates of deposition of particles by thermophoresis. We shall review briefly all the calculations of deposition rates in particular flow systems that are known to us, and will add some new exact solutions. Interpretation and comparison of the results for all these special cases allows the inference of some general conclusions which we suggest may be applied in cases lying outside the reach of direct computation.

\section{GENERAL FORMULATION OF THE PROBLEM OF CALCULATING THE DEPOSITION RATE}

The linear relation between the thermophoretic velocity of a particle and the local temperature gradient is usually written as

$$
\mathbf{V}=-K \frac{\nu}{T} \nabla T
$$

where $T$ is the ambient absolute temperature of the gas, $\nu$ is the kinematic viscosity of the gas, and the thermophoretic coefficient $K$ depends on the properties of both the gas and the particle. Theoretical expressions for $K$ are available only for large or small values of the Knudsen number $\lambda / a$. A summary of the theory of thermophoresis and the correspondence with observations of particle movement may be found in the recent paper by Talbot, Cheng, Schefer and Willis (4). These authors give evidence in support of their belief that the following expression for $K$ for a spherical particle of radius $a$ is tolerably accurate for all regimes from free molecular to continuum flow:

$$
K=2 C_{\mathrm{s}} \frac{\left(k_{\mathrm{g}} / k_{\mathrm{p}}+C_{\mathrm{t}} \lambda / a\right)\left[1+\frac{\lambda}{a}\left(1.2+0.41 e^{-0.88 a / \lambda}\right)\right]}{\left(1+3 C_{\mathrm{m}} \lambda / a\right)\left(1+2 k_{\mathrm{g}} / k_{\mathrm{p}}+2 C_{\mathrm{t}} \lambda / a\right)}
$$

where $\lambda$ is the mean free path of molecules in the gas, $C_{\mathrm{s}}, C_{\mathrm{t}}, C_{\mathrm{m}}$ are the thermal creep coefficient, temperature jump coefficient, and velocity jump coefficient, respectively, and have values $C_{\mathrm{s}}=1.147, C_{\mathrm{t}}=2.20, C_{\mathrm{m}}$ $=1.146$ for complete accommodation (see, for example, Shen (5)), and $k_{\mathrm{g}}$ and $k_{\mathrm{p}}$ are the thermal conductivities of the gas and the particle. Figure 1 shows $K$ as a function of the Knuden number $\lambda / a$, with $k_{\mathrm{g}} / k_{\mathrm{p}}$ as a parameter, according to [2.2]. Accurate measurement of $K$ is difficult and the agreement between the available data and the theoretical values is not close (see Derjaguin et al. (6)). In the literature concerned with calculation of the rate of thermophoretic deposition $K$ is usually taken as ranging from 0.2 to 1.2 .

The gas viscosity $\nu$ appears in [2.1] essentially as a measure of the product of the mean free path and the mean molecular speed, and any other diffusivity of the gas would serve as well. Inasmuch as the thermal diffusivity $\alpha=k_{\mathrm{g}} / \rho c_{\mathrm{p}}$ enters into a calculation of thermophoretic deposition rates whenever the temperature distribution in the gas is determined as a part of the problem (instead of simply being given), whereas the kinematic viscosity $\nu$ does so only if the velocity distribution also is determined, the replacement

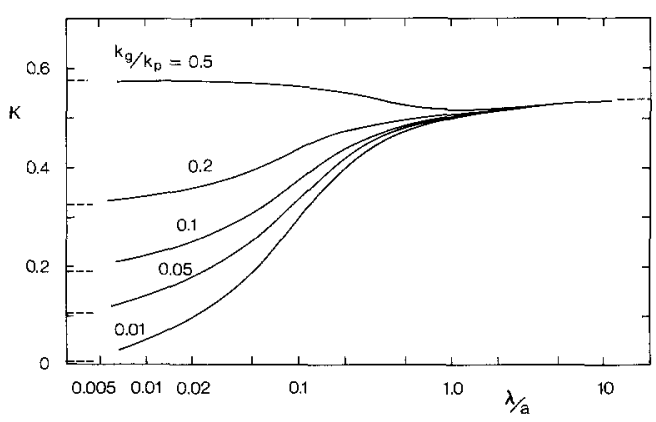

FIG. 1. Values of the thermophoretic coefficient $K$ for a spherical particle of radius a according to the expression [2.2] proposed by Talbot, Cheng, Schefer, and Willis (4). $\lambda$ is the mean free path of the gas molecules and $k_{\mathrm{g}} / k_{\mathrm{p}}$ is the ratio of the thermal conductivity of the gas to that of the particle. 
of $\nu$ by $\alpha$ in [2.1] results in some simplification in the analysis, especially for flow fields such as Poiseuille flow in a tube for which the form of the velocity distribution is independent of $\nu$. We shall therefore rewrite [2.1] as

$$
\mathbf{V}=-H \frac{\alpha}{T} \nabla T
$$

where the new thermophoretic coefficient $H$ is related to the more conventional one by

$$
H=\sigma K .
$$

The Prandtl number $\sigma \equiv \nu / \alpha$ is equal to 0.72 for air and lies within the range $0.66-0.80$ for common gases at normal temperatures and pressures, so the numerical consequences of this change of definition are not great. Note that since the values of $k_{\mathrm{g}}$ and $\sigma$ vary slowly with the temperature of a gas, it is desirable to avoid the assumption that $H$ is constant in a problem of thermophoretic deposition involving large temperature differences.

We shall assume that the particle velocity due to external body forces is negligible, and also that the particle volume fraction is so small that the velocity and temperature distributions in the gas are unaffected by the presence of the particles. These assumptions are valid for common aerosols unless a strong electric field is applied to charged particles.

The distribution of particle concentration $C$ (= number density) is affected in general by convection, diffusion, and thermophoresis, and is determined by the equation

$$
\frac{\partial C}{\partial t}+\mathbf{U} \cdot \nabla C=\nabla \cdot(D \nabla C)-\nabla \cdot(C \mathbf{V}),[2.5]
$$

where $\mathbf{U}$ is the local gas velocity and $\mathbf{V}$ is given by [2.3]. The gas temperature field $T$ is governed by the equation

$$
\frac{\partial T}{\partial t}+\mathbf{U} \cdot \nabla \mathrm{T}=\nabla \cdot(\alpha \nabla \mathrm{T})
$$

in which the dissipation due to viscosity has been neglected. The gas velocity appearing in [2.5] and [2.6] will be regarded as given.
It is convenient to introduce a length $L$ and speed $U_{0}$ which are characteristic of the flow field and to make the position vector, time, and the flow velocity nondimensional using $L$ and $U_{0}$. Both $C$ and $T$ appear homogeneously in [2.3], [2.5], and [2.6] and so can be nondimensionalized when necessary by dividing by representative values $C_{0}$ and $T_{0}$ without affecting the form of the equations. Equations [2.5] and [2.6] can now be written as

$$
\begin{aligned}
\frac{\partial C}{\partial \tau}+\mathbf{u} \cdot \nabla C=\frac{1}{\complement}[ & \nabla \cdot\left(\frac{D}{\alpha_{0}} \nabla C\right) \\
& \left.+\nabla \cdot\left(\frac{C H \alpha}{T \alpha_{0}} \nabla T\right)\right] \\
\frac{\partial T}{\partial \tau}+\mathbf{u} \cdot \nabla T & =\frac{1}{Ð} \nabla \cdot\left(\frac{\alpha}{\alpha_{0}} \nabla T\right),
\end{aligned}
$$

where $\tau=t U_{0} / L, \mathbf{u}=\mathbf{U} / U_{0}, \mathbb{P}=U_{0} L / \alpha_{0}$, and $\alpha_{0}$ is a representative value of $\alpha$ (which may vary with temperature). The Peclet number $\mathbb{P}$ depends on the flow system and on the gas properties; for guidance on magnitudes we note that $\mathbb{Q}=5 \times 10^{2}$ for $U_{0}=1$ $\mathrm{m} / \mathrm{s}, L=10 \mathrm{~mm}$ and $\alpha_{0}=20 \mathrm{~mm}^{2} / \mathrm{s}$ (as for air at $15^{\circ} \mathrm{C}$ ). Values of $(\mathbb{D}$ much larger than unity are common in practice.

As stated earlier, $D$ is very much smaller than any of the gas diffusivities such as $\alpha$. Hence, provided the temperature differences are not extremely small by comparison with the absolute temperature, the first term within the brackets on the right-hand side of Eq. [2.7] can be neglected except in a very thin concentration boundary layer in the vicinity of a wall, and [2.7] reduces to

$$
\frac{\partial C}{\partial t}+\mathbf{u} \cdot \nabla C=\frac{1}{\rrbracket} \nabla \cdot\left(\frac{C H \alpha}{T \alpha_{0}} \nabla T\right),
$$

which is a useful simplification of one of the governing equations.

It is usually not necessary to consider conditions within the thin concentration boundary layer in order to find the rate of the deposition at a rigid boundary because 
the gas velocity relative to the boundary is correspondingly small there. Estimates of the thickness of the concentration boundary layer depend on the nature of the flow system, but they all contain a positive power of $D$ so that the ratio of the gas velocity in the concentration boundary layer to the thermophoretic velocity approaches zero as $D / \alpha \rightarrow 0$. Thus when $D / \alpha \ll 1$ the particles that enter the concentration boundary layer by thermophoresis continue across the boundary layer to the wall without significant change of their downstream position, as shown with more analytical detail by Walker, Homsy, and Geyling (7) for the case of flow in a tube with a sudden change of temperature at one section of the tube. Hence the local flux of particles to the wall due to thermophoresis is given by

$$
J=-C_{\mathrm{w}} \mathbf{n} \cdot \mathbf{V}_{\mathrm{w}}=H \alpha C_{\mathrm{w}}\left[\frac{\mathbf{n} \cdot \nabla T}{T}\right]_{\mathrm{w}},
$$

where the suffix $w$ denotes the value at the wall and in the case of $C_{w}$ it is understood that $C_{\mathrm{w}}$ is determined from equations in which $D=0$. The unit normal to the wall, $\mathbf{n}$, is directed into the fluid. ${ }^{1}$ We may also write [2.10] as

$$
J=H(C / T)_{w} F, \quad F=\alpha(\mathbf{n} \cdot \nabla T)_{w}
$$

where $\rho c_{\mathrm{p}} F$ is the local density of heat flux to the wall.

There is a further contribution to the deposition rate due to Brownian diffusion, viz., $D(\mathbf{n} \cdot \nabla C)_{\mathrm{w}}$, but owing to the smallness of $D$ this contribution is normally much smaller than that due to thermophoresis.

The nature of the problem of calculation of the thermophoretic deposition rate is now clear. Provided the velocity field is known,

\footnotetext{
${ }^{1}$ Formula [2.10] is of course applicable only to the situation in which the wall is colder than the adjacent gas and the thermophoretic velocity is towards the wall. The distribution of concentration shows very different features when the wall is hotter than the gas (see Goren (8)).
}

Journal of Colloid and Interface Science, Vol. 107, No. 1, September 1985 the temperature distribution may be determined from Eq. [2.8] and the imposed boundary conditions on the temperature. Equation [2.9] for the particle concentration may then be solved. Finally the local rate of deposition of particles at the wall is found from [2.10] and the total rate of deposition by integration over the boundary.

It will be noticed that only temperature differences occur in [2.8], whereas the absolute temperature also occurs in [2.9]. Thus, for given geometry of the boundaries the concentration $C$ depends on $H, \mathbb{P}$, and $\Delta T /$ $T_{0}$, where $\Delta T$ is representative of the temperature differences. An alternative form of this temperature parameter is $T_{1} / T_{0}$, where $T_{1}$ and $T_{0}$ are temperatures specified by the boundary conditions (so that $\Delta T$ can be chosen as $T_{0}-T_{1}$ ). It will often be possible to eliminate $P$ from the equations by a scale transformation, although the precise way in which this may be done depends on the form of the flow field. However, the other two parameters, $H$ and $T_{1} / T_{0}$, have a significant influence on the structure of the solution.

There are two main types of practical problem of thermophoretic deposition. In one type a stream of gas containing suspended particles flows past a body or group of bodies held at a lower temperature than that of the oncoming stream, and particles are deposited on the body surfaces. In the other type the gas is flowing inside a tube whose wall temperature is lower than that of the oncoming fluid and the rate of deposition on the interior tube wall is to be calculated. In both types of problem it will often happen that $T$ and $C$ are uniform in the fluid far upstream (with values $T_{0}$ and $C_{0}$ say) and decrease monotonically along particle trajectories which originate in the upstream reservoir and terminate at a cold boundary. Specification of the value of $C$ in the upstream fluid is a sufficient boundary condition for the determination of $C$, but more boundary conditions on $T$ are needed since the order of the differential equation for $T$ is one higher than that for $C$. 
Specific cases of both types of problem will be considered. However, we first establish some general results applicable to a variety of flow systems which will prove to be useful for the interpretation and exploitation of these specific cases.

\section{SOME GENERAL RESULTS APPLICABLE TO A VARIETY OF FLOW SYSTEMS}

\section{An Exact Solution for $H=1$}

It is evident that when $H=1$ the two governing equations [2.8] and [2.9] allow the simple relation between the two dependent variables

$$
C / T=\text { const } .
$$

Provided that the boundary conditions are compatible with this relation, as they are if the only boundary condition on $C$ is that $C$ $=C_{0}$ everywhere far upstream and if $T$ also is uniform there (and equal to $T_{0}$ ), the required solution for $C$ is

$$
C / C_{0}=T / T_{0},
$$

with $T$ being determined from [2.8]. The distributions of temperature and concentration are here identical and the local rate of deposition of particles at a wall follows from [2.11] as

$$
J=C_{0} F / T_{0}
$$

where $F$ is the heat flux density at the wall.

The generality of this simple exact solution seems not to have been commented on hitherto, but the fact that the equations have the solution [3.2] when $H=1$ has been noticed by Goren (8) in the case of flow in the Blasius boundary layer on a cooled flat plate and by Weinberg (9) in the case of flow in a circular tube whose wall temperature has a discontinuous jump to a smaller constant value. Values of $H$ near unity are not unknown in practice, so that the solution has some direct value. We shall also find it useful as a guide when formulating approximations for arbitrary values of $H$.

\section{Bounds on the Value of $C$ at the Cold Wall}

An analytical solution for $C$ is unlikely to exist when $H \neq 1$, but it is possible to find upper and lower bounds on the value of $C$ which coincide in the case $H=1$. We begin by combining Eqs. [2.8] and [2.9] in two different ways to give

$$
\begin{gathered}
\left\{\frac{\partial}{\partial \tau}+\left(\mathbf{u}-\frac{H \alpha}{\rrbracket \alpha_{0}} \frac{\nabla T}{T}\right) \cdot \nabla\right\} \log \frac{C}{T} \\
=-\frac{1-H}{Ð T} \nabla \cdot\left(\frac{\alpha}{\alpha_{0}} \nabla T\right) \\
+\frac{\alpha}{\alpha_{0} \rrbracket T} \nabla T \cdot \nabla H
\end{gathered}
$$

$$
\begin{gathered}
\left\{\frac{\partial}{\partial \tau}+\left(\mathbf{u}-\frac{H \alpha}{\mathbb{D} \alpha_{0}} \frac{\nabla T}{T}\right) \cdot \nabla\right\} \log \frac{C}{T^{H}} \\
=-\frac{H(1-H) \alpha}{\mathbb{P} \alpha_{0}} \frac{|\nabla T|^{2}}{T^{2}} \\
+\frac{\alpha}{\alpha_{0} \mathbb{P} T} \nabla T \cdot \nabla H .
\end{gathered}
$$

The operator on each of the two left-hand sides represents the time derivative following a particle whose velocity is the resultant of that due to convection with the fluid and that due to thermophoresis. Each particle trajectory originates (so we assume) at "infinity" upstream where $C=C_{0}$ and $T=T_{0}$, and we are interested in those trajectories that terminate at a boundary where the temperature is held at a value smaller than $T_{0}$.

The signs of the right-hand sides of [3.4] and [3.5] are now determined by inspection. The sign of $\nabla \cdot\left(\left(\alpha / \alpha_{0}\right) \nabla T\right)$ is the same as that of $(\partial / \partial \tau+\mathbf{u} \cdot \nabla) T$, which will be negative in all those common cases in which the streamlines approach or pass near to a boundary whose temperature never increases in the flow direction. Thus since $H$ is a slowly-varying function of $T$ it follows from [3.4] that $C / T$ increases along a particle trajectory when $H<1$ (the typical case) and decreases when $H>1$. The sign of the first (and dominant) term on the right-hand side 
of [3.5] is unambiguously negative when $H$ $<1$, showing that $C / T^{H}$ decreases along $a$ particle trajectory.

Thus the value of $C$ at a cold boundary, where $T=T_{1}$, satisfies the inequalities

$$
\frac{T_{1}}{T_{0}}<\frac{C_{1}}{C_{0}}<\left(\frac{T_{1}}{T_{0}}\right)^{H}
$$

when $H<1$. As $H \rightarrow 1$ the exact solution described above is recovered.

\section{An Approximate Solution for the Concentration}

It may be seen that, if $\Delta T$ is representative of the temperature differences in the fluid, the right-hand sides of both [3.4] and [3.5] approach zero as $\Delta T / T_{0} \rightarrow 0$. This indicates that both $C / T$ and $C / T^{H}$ are approximately constant along a particle trajectory when $\Delta T /$ $T_{0} \ll 1$ because $T$ is then approximately constant. More significant is the fact that the right-hand side of [3.5] is of order $\left(\Delta T / T_{0}\right)^{2}$ when $\Delta T / T_{0} \ll 1$, from which we learn that

$$
\frac{C}{C_{0}}=1-H\left(\frac{T_{0}-T}{T_{0}}\right)+\mathrm{O}\left(\frac{\Delta T^{2}}{T_{0}^{2}}\right)
$$

when $\Delta T / T_{0} \ll 1$.

Equation [3.5] shows also that the error term in [3.7] is negative when $H<1$ and is zero in the cases $H=1$ and $H=0$. A more accurate representation of the particle concentration than [3.7] is

$$
\frac{C}{C_{0}}=\frac{T}{T_{0}}\left\{1+(1-H)\left(\frac{T_{0}-T}{T_{0}}\right)\right\},
$$

which coincides with [3.7] to order $\Delta T / T_{0}$, is exact to all orders when $H=1$, and gives values of $C / C_{0}$ which are greater than $T / T_{0}$ but less than $\left(T / T_{0}\right)^{H}$. The expression [3.8] is of course not unique in having these properties.

\section{Conjecture that the Level Surfaces of $C$ and $T$ Coincide Approximately}

It has been seen that

$$
C / C_{0}=\left(T / T_{0}\right)^{H}
$$

Journal of Colloid and Interface Science, Vol. 107, No. 1, September 1985 is a solution of the governing equations when $H=1$ (and in the trivial case $H=0$ ) and also, correct to order $\Delta T / T_{0}$, when $\Delta T / T_{0}$ $\ll 1$ for arbitrary $H$. We have also found upper and lower bounds on $C$ which depend on position only through their dependence on $T$, in cases in which all particle trajectories originate in a reservoir where $C$ and $T$ are uniform. All these deductions are compatible with the level surfaces of $C$ and $T$ being coincident throughout the fluid. There is no reason to suppose an exact coincidence in general, but the fact that the molecular flux densities of $C$ and $T$ are everywhere parallel, and that the ratio of the magnitudes of these flux vectors is constant when $H=1$ or 0 , suggests an approximate coincidence. The question is, does the assumption of a general relationship between $C$ and $T$ have sufficient accuracy for some practical purposes. As to the form of such a general approximate relationship, [3.8] is clearly a candidate.

It would be very valuable if there were a relationship between $C$ and $T$ for flow fields of complex form, because the total rate of deposition of particles on a wall of uniform temperature would then be proportional to the total heat flux to the wall. The integral of the particle density flux over the cold wall is given by [2.11] as

$$
\int J d A=\int\left(H \frac{C}{T}\right)_{\mathrm{w}} F d A,
$$

and in a case in which $T$ and $C$ have the uniform values $T_{1}$ and $C_{1}$ at the wall this reduces to

$$
\int J d A=H \frac{C_{1}}{T_{1}} \int F d A
$$

The total heat flux to the wall is more readily measured than the particle flux, and if $\int F d A$ is known determination of $\int J d A$ requires only the value of $C_{1}$, which may be found from the relation between $T$ and $C$. Empirical knowledge of the heat transfer from bodies held at a uniform temperature in a stream of fluid could thus be used to estimate the 
total rate of deposition of particles on the body surface.

It is worthwhile therefore to use the available analytical or numerical data as a test of the conjecture that the level surfaces of $C$ and $T$ coincide approximately, and we do this in the next three sections.

Figure 2 shows the information gathered in this section concerning the value of the particle concentration at a cold isothermal boundary where the temperature is $T_{1}$, for the particular case $H=0.3$. The simple expression for $C_{1} / C_{0}$ suggested in [3.8] is seen to lie roughly midway between the upper and lower bounds, except that when $\left(T_{0}-T_{1}\right) / T_{0} \ll 1$ it coincides with the upper bound and with the exact asymptote [3.7]. For values of $H$ closer to 1.0 the upper and lower bounds are closer.

4. EXACT SOLUTION FOR THE PARTICLE CONCENTRATION IN STEADY BOUNDARYLAYER FLOW PAST A COLD FLAT PLATE, CYLINDER, OR BODY OF REVOLUTION

Solutions for the temperature distributions in gas of temperature $T_{0}$ flowing steadily past a flat plate held at temperature $T_{1}$ and in the region near the forward stagnation point on a cylinder or body of revolution of temperature $T_{1}$ in a stream for $\mathbb{D} \gg 1$ are well known (see Goldstein (10) for the flat plate and cylinder and Sibulkin (11) for the body of revolution). These three boundary-layer solutions for $T$, which are exact aside from the fact that the kinematic viscosity and the Prandtl number are taken to be uniform over the fluid, are members of the same mathematical family, as are the corresponding velocity distributions. Goren (8) has shown that it is possible also to find an exact solution to the equation for the particle concentration in the case of flow past a flat plate and Homsy, Geyling and Walker (12) later solved numerically the equation for the concentration in the boundary layer near the stagnation point on a cylinder. We give here an integrated description of the solution for the particle concentration in all three cases, that for the flow past a body of revolution being new.

The velocity boundary layers in all three flow systems have a similarity form, and may be described by stream functions defined as
(a) flat plate
$\psi=\left(\nu U_{0} x\right)^{1 / 2} f(\eta)$
$\eta=\frac{1}{2}\left(\frac{U_{0}}{\nu x}\right)^{1 / 2} y$,
(b) cylinder
$\psi=(\beta \nu)^{1 / 2} x f(\eta)$,
$\eta=\left(\frac{\beta}{\nu}\right)^{1 / 2} y$,
$\psi=\left(\frac{1}{2} \beta \nu\right)^{1 / 2} x^{2} f(\eta)$
$\eta=\left(\frac{2 \beta}{\nu}\right)^{1 / 2} y$,

where $x$ represents streamwise distance along the surface from the leading edge or stagnation point on the body, $y$ is the position coordinate normal to the boundary, $U_{0}$ is the speed of the stream flowing past the flat plate, and $\beta x$ is the speed of the fluid at the outer edge of the boundary layer near the stagnation point on the cylinder or body of revolution. The equations and boundary conditions for the stream functions are
(a) flat plate
$f^{\prime \prime \prime}=-f f^{\prime \prime}$,
$f(0)=0, \quad f^{\prime}(0)=0, \quad f^{\prime}(\infty)=1$,
(b) cylinder
$f^{\prime \prime \prime}=-f f^{\prime \prime}+f^{\prime 2}-1$,
$f(0)=0, \quad$
$f^{\prime}(0)=0, \quad f^{\prime}(\infty)=1$,
(c) body of revolution
$f^{\prime \prime \prime}=-f f^{\prime \prime}+\frac{1}{2}\left(f^{2}-1\right)$
$f(0)=0$,
$f^{\prime}(0)=0, \quad f^{\prime}(\infty)=1$. 
Tables of the solution for $f$ in each case are available (seeGoldstein (10) and Schlichting (13)).

The equation for the temperature $T$ has a solution which is a function of $\eta$ alone. With the particular definitions of $\psi$ and $\eta$ shown in [4.1] this is the same equation in all three cases, viz.,

$$
\begin{gathered}
T^{\prime \prime}+\sigma f T^{\prime}=0, \\
T(0)=T_{1}, \quad T(\infty)=T_{0},
\end{gathered}
$$

where $\sigma$ denotes the Prandtl number $\nu / \alpha$, here assumed constant. The solution of Eq. [4.3] is

$\frac{T-T_{1}}{T_{0}-T_{1}}$

$$
=\gamma \int_{0}^{\eta} \exp \left(-\sigma \int_{0}^{\xi} f(\zeta) d \zeta\right) d \xi
$$

where the constant $\gamma$ is given by

$$
\begin{array}{r}
\gamma=\left[\int_{0}^{\infty} \exp \left(-\sigma \int_{0}^{\xi} f(\zeta) d \zeta\right) d \xi\right]^{-1} \\
=\frac{1}{T_{0}-T_{1}}\left[\frac{\partial T}{\partial \eta}\right]_{\eta=0} .
\end{array}
$$

Numerical evaluation of $f(\eta)$ and thence of $\gamma$ has shown that $\gamma$ is represented well by $0.664 \sigma^{1 / 3}(=0.595$ for air) in the case of the flat plate (Goldstein (10), p. 624), by $0.570 \sigma^{0.4}$ $(=0.500$ for air) in the case of the cylinder (Goldstein (10), p. 632), and by $0.540 \sigma^{0.4}$ $(=0.473$ for air) in the case of the body of revolution (Sibulkin (11)).

We likewise find that the steady-state form of Eq. [2.9] for the particle concentration admits a solution which is a function of $\eta$ alone. This function satisfies the equation

$$
\left(\frac{T^{\prime}}{T}+\frac{f}{H}\right) C^{\prime}+\left(\frac{T^{\prime \prime}}{T}-\frac{T^{2}}{T^{2}}\right) C=0
$$

( $H$ being assumed constant here) with the boundary condition $C(\infty)=C_{0}$. The solution is

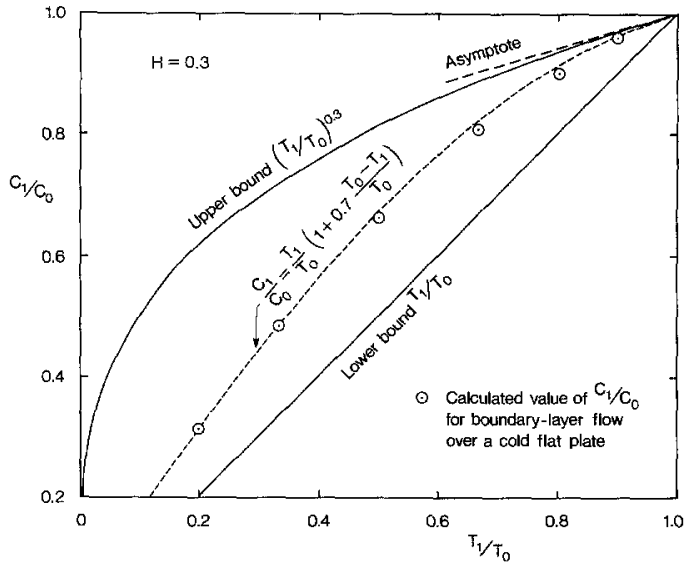

FiG. 2. Values of the particle concentration $C_{1}$ at a cold isothermal boundary where the temperature is $T_{1}$ in the case $H=0.3$. The two solid curves show the bounds [3.6], the long-dashed line is the exact asymptote [3.7], and the short-dashed line is the approximate relation [3.8]. The encircled points represent the exact result [4.8] for boundary-layer flow of air over a cold flat plate.

$$
\frac{C}{C_{0}}=\exp \left(-\int_{\eta}^{\infty} \frac{\left(\frac{T^{\prime}}{T}\right)^{2}-\frac{T^{\prime \prime}}{T}}{\frac{T^{\prime}}{T}+\frac{f}{H}} d \eta\right)
$$

in which $T$ is given by [4.4].

It is a feature of these similarity solutions that $T$ and $C$ are functions of $\eta$ alone, implying a relationship between $T$ and $C$. At the body surface $(\eta=0)$, where $T$ has the constant value $T_{1}, C$ is also constant, with value $C_{1}$ given by

$$
\frac{C_{1}}{C_{0}}=\exp \left(-\int_{0}^{\infty} \frac{\left(\frac{T^{\prime}}{T}\right)^{2}-\frac{T^{\prime \prime}}{T}}{\frac{T^{\prime}}{T}+\frac{f}{H}} d \eta\right)
$$

Using the tables of $f$ given in the literature one can now calculate $T$ numerically from [4.4], and then $C$ from [4.7], as functions of $\eta$ for various values of $\sigma, T_{1} / T_{0}$, and $H$. We give in Table I the values of $C / C_{0}$ at the body surface for the case of air $(\sigma=0.72)$ 
for each of the three flow fields. Despite the physical differences between the three flow fields, the three values of $C_{1} / C_{0}$ are close to each other. We note also from the table that the simple expression [3.8] agrees with these calculated values of $C_{1} / C_{0}$ to within a few percent, except when $H$ and $T_{1} / T_{0}$ are both small. The values of $C_{1} / C_{0}$ for the flat plate in the case $H=0.3$ are shown in Fig. 2 for comparison with [3.8].

The local particle flux density at the body surface is seen from [2.11] and [4.5] to be given by

$$
\begin{aligned}
\frac{J}{C_{0}} & =H \frac{C_{1}}{C_{0}} \frac{F}{T_{1}} \\
& =H \alpha \gamma \frac{C_{1}}{C_{0}} \frac{T_{0}-T_{1}}{T_{1}}\left(\frac{\partial \eta}{\partial y}\right)_{y=0}
\end{aligned}
$$

where $\gamma$ and $C_{1} / C_{0}$ vary slightly between the three flow fields and are known numerically, $\gamma$ as a function of $\sigma$ and $C_{1} / C_{0}$ as a function of $\sigma, H$ and $T_{1}{ }^{*} / T_{0}$. On substituting for $\partial \eta / \partial y$ from [4.1] we finally obtain the following explicit expressions for the particle flux density for each of the three flow fields:

$$
\left.\begin{array}{ll}
\text { (a) flat plate } & J=\frac{1}{2} H C_{1} \alpha \gamma \frac{T_{0}-T_{1}}{T_{1}}\left(\frac{U_{0}}{\nu x}\right)^{1 / 2} \\
\text { (b) cylinder } & J=H C_{1} \alpha \gamma \frac{T_{0}-T_{1}}{T_{1}}\left(\frac{\beta}{\nu}\right)^{1 / 2} \\
\text { (c) body of revolution } & J=H C_{1} \alpha \gamma \frac{T_{0}-T_{1}}{T_{1}}\left(\frac{2 \beta}{\nu}\right)^{1 / 2} \cdot
\end{array}\right\}
$$

Empirical values of the parameter $\beta$ in the cases of flow at large Reynolds number past a circular cylinder of radius $a$ and a sphere of radius $a$ are not far from the values $2 U_{0} /$ $a$ and $3 U_{0} / 2 a$ respectively corresponding to wholly irrotational flow.

\section{BLASIUS SERIES FOR STEADY FLOW PAST A CYLINDER}

The solutions described in Section 4 for the fluid velocity, temperature, and particle concentration in the boundary layer near the forward stagnation point on a cylinder in stream have been extended to larger distances downstream by means of the so-called Blasius series. It was pointed out by Blasius in 1908 that, if the fluid velocity at the outer edge of the boundary layer is written as a power series, viz.,

$U(x)=U_{1} x+U_{3} x^{3}+U_{5} x^{5}+\cdots+,[5.1]$

where $x$ is the distance along the surface of a symmetrical cylinder from the stagnation point, the stream function for the flow in the boundary layer may likewise be expressed as a series

$$
\begin{gathered}
\psi(x, y)=\left(\frac{\nu}{U_{1}}\right)^{1 / 2}\left(U_{1} x f_{1}+4 U_{3} x^{3} f_{3}\right. \\
\left.+6 x^{5}\left\{U_{5} f_{51}+\frac{U_{3}^{2}}{U_{1}} f_{52}\right\}+\cdots+\right)
\end{gathered}
$$

in which $f_{1}, f_{3}, f_{51}, f_{52}, \ldots$, are functions of the similarity variable $\eta\left(=y\left(U_{1} / \nu\right)^{1 / 2}\right.$ - see [4.1]) which are universal in the sense of being independent of $U_{1}, U_{3}, U_{5}, \ldots$ The coefficient $U_{1}$ here replaces the symbol $\beta$ used in Section 4 and $f_{1}$ replaces $f$. The functions $f_{1}, f_{3}, f_{51}, f_{52}, \ldots$, satisfy ordinary differential equations which can be solved numerically, and the solutions have been tabulated in textbooks (e.g., Schlichting (13)).

It has also been known for many years that the temperature in the thermal boundary layer at the surface of a cylinder held at a temperature $T_{1}$ different from the tempera- 
TABLE I

Values of the Particle Concentration Ratio $C_{1} / C_{0}$ at the Body Surface Where the Temperature is $T_{1}$

\begin{tabular}{|c|c|c|c|c|c|c|c|}
\hline \multirow[b]{2}{*}{$H$} & \multicolumn{7}{|c|}{$T_{1} / T_{0}$} \\
\hline & & 0.2 & 0.3 & 0.5 & $0.6^{2}$ & 0.8 & 0.9 \\
\hline 1.0 & & 0.2 & 0.3 & 0.5 & $0 . \dot{6}$ & 0.8 & 0.9 \\
\hline \multirow[t]{5}{*}{0.8} & (a) & 0.2190 & 0.3606 & 0.5322 & 0.6970 & 0.8234 & 0.9142 \\
\hline & (b) & 0.2203 & 0.3624 & 0.5341 & 0.6986 & 0.8244 & 0.9147 \\
\hline & (c) & 0.2199 & 0.3618 & 0.5334 & 0.6981 & 0.8241 & 0.9145 \\
\hline & (d) & 0.2186 & 0.3599 & 0.5314 & 0.6965 & 0.8230 & - \\
\hline & (e) & 0.2320 & 0.3778 & 0.5500 & 0.7111 & 0.8320 & 0.9180 \\
\hline \multirow[t]{5}{*}{0.7} & (a) & 0.2308 & 0.3772 & 0.5510 & 0.7142 & 0.8362 & 0.9218 \\
\hline & (b) & 0.2330 & 0.3800 & 0.5541 & 0.7167 & 0.8378 & 0.9225 \\
\hline & (c) & 0.2323 & 0.3791 & 0.5531 & 0.7159 & 0.8373 & 0.9223 \\
\hline & (d) & 0.2300 & 0.3760 & 0.5499 & 0.7134 & 0.8356 & - \\
\hline & (e) & 0.2480 & 0.4000 & 0.5750 & 0.7333 & 0.8480 & 0.9270 \\
\hline \multirow[t]{5}{*}{0.5} & (a) & 0.2616 & 0.4190 & 0.5971 & 0.7545 & 0.8650 & 0.9384 \\
\hline & (b) & 0.2661 & 0.4248 & 0.6028 & 0.7588 & 0.8676 & 0.9393 \\
\hline & (c) & 0.2647 & 0.4230 & 0.6010 & 0.7575 & 0.8668 & 0.9389 \\
\hline & (d) & 0.2599 & 0.4169 & 0.5950 & 0.7530 & 0.8642 & - \\
\hline & (e) & 0.2800 & 0.4444 & 0.6250 & 0.7778 & 0.8800 & 0.9450 \\
\hline \multirow[t]{5}{*}{0.3} & (a) & 0.3107 & 0.4823 & 0.6618 & 0.8068 & 0.9000 & 0.9568 \\
\hline & (b) & 0.3194 & 0.4925 & 0.6711 & 0.8133 & 0.9036 & 0.9582 \\
\hline & (c) & 0.3166 & 0.4893 & 0.6682 & 0.8113 & 0.9025 & 0.9578 \\
\hline & (d) & 0.3078 & 0.4788 & 0.6587 & 0.8048 & 0.8989 & - \\
\hline & (e) & 0.3120 & 0.4889 & 0.6750 & 0.8222 & 0.9120 & 0.9630 \\
\hline \multirow[t]{5}{*}{0.15} & (a) & 0.3807 & 0.5650 & 0.7380 & 0.8623 & 0.9339 & 0.9735 \\
\hline & (b) & 0.3953 & 0.5805 & 0.7505 & 0.8700 & 0.9377 & 0.9749 \\
\hline & (c) & 0.3907 & 0.5756 & 0.7466 & 0.8676 & 0.9365 & 0.9744 \\
\hline & (d) & - & - & - & - & - & - \\
\hline & (e) & 0.3360 & 0.5222 & 0.7125 & 0.8555 & 0.9360 & 0.9765 \\
\hline \multirow[t]{5}{*}{0.10} & (a) & 0.4226 & 0.6106 & 0.7761 & 0.8875 & 0.9481 & 0.9800 \\
\hline & (b) & 0.4406 & 0.6285 & 0.7895 & 0.8953 & 0.9518 & 0.9813 \\
\hline & (c) & 0.4349 & 0.6229 & 0.7853 & 0.8929 & 0.9507 & 0.9809 \\
\hline & (d) & - & - & - & - & - & - \\
\hline & (e) & 0.3440 & 0.5333 & 0.7250 & 0.8667 & 0.9440 & 0.9810 \\
\hline
\end{tabular}

Note. The five numbers in each section of the table refer to: (a) the boundary layer on a flat plate in a stream of air; the boundary layer near the stagnation point (b) on a cylinder and (c) on a body of revolution; (d) Poiseuille flow in a tube at small distances downstream from a sudden drop in wall temperature; and (e) the value given by the relation [3.8].

ture $T_{0}$ of the oncoming stream may be written as

$\frac{T-T_{1}}{T_{0}-T_{1}}=\theta_{0}+\frac{U_{3}}{U_{1}} x^{2} \theta_{2}$

$$
+x^{4}\left(\frac{U_{5}}{U_{1}} \theta_{41}+\frac{U_{3}^{2}}{U_{1}^{2}} \theta_{42}\right)+\cdots+
$$

in which $\theta_{0}, \theta_{2}, \theta_{41}, \theta_{42}, \ldots$, are functions of $\eta$ and of the Prandtl number $\sigma$ which 
satisfy differential equations involving the functions $f_{1}, f_{3}, f_{51}, f_{52}, \ldots$ Values of all the functions involved in terms up to $x^{10}$ have been calculated and published by Newman (14).

The natural next step of writing the particle concentration as

$$
\begin{aligned}
\frac{C}{C_{0}} & =\Phi_{0}+\frac{U_{3}}{U_{1}} x^{2} \Phi_{2} \\
& +x^{4}\left(\frac{U_{5}}{U_{1}} \Phi_{41}+\frac{U_{3}^{2}}{U_{1}^{2}} \Phi_{42}\right)+\cdots+
\end{aligned}
$$

where $C_{0}$ is the concentration everywhere far upstream of the cylinder and $\Phi_{0}, \Phi_{2}, \Phi_{41}$, $\Phi_{42}, \ldots$, are functions of $\eta, \sigma$, and $H\left(\Phi_{0}\right.$ being given by [4.7]), was taken recently by Homsy, Geyling and Walker (12). These authors completed the formidable calculation of all the functions needed for the determination of the coefficient of $x^{4}$ in [5.4], and although they do not give tables of these functions in their paper they give the values of the coefficients in the corresponding series for the particle flux density at the surface of the cylinder, viz.,

$$
\begin{aligned}
J= & C_{0} H \alpha\left(\frac{U_{1}}{\nu}\right)^{1 / 2}\left(J_{0}+\frac{U_{3}}{U_{1}} x^{2} J_{2}\right. \\
& \left.+x^{4}\left\{\frac{U_{5}}{U_{1}} J_{41}+\frac{U_{3}^{2}}{U_{1}^{2}} J_{42}\right\}+\cdots+\right)
\end{aligned}
$$

for $\sigma=0.71$ and a limited number of values of $H$ and $T_{1} / T_{0}$. The value of $J_{0}$ is already known from [4.10].

These values of $J_{0}, J_{2}, J_{41}$, and $J_{42}$ provided by Homsy, Geyling, and Walker are valuable inasmuch as they make possible the accurate calculation of the rate of deposition of particles over quite a large part of the surface of a cylinder of any (symmetrical) shape in a stream, in fact over most of the part of the surface at which there is an attached laminar boundary layer. And, as the authors point out, this may be where nearly all the deposition takes place.

Our interest in the results of Homsy, Geyling, and Walker here is different, however. Once the heat flux density at the cylinder surface is known-and for this we have Newman's tables-Homsy, Geyling, and Walker's calculation of the particle flux is, in effect, a calculation of the value of the concentration at the surface. Formally we have from [2.11], [5.3], and [5.5]

$$
\begin{aligned}
\frac{C_{1}}{C_{0}} & =\frac{J T_{1}}{H F C_{0}} \\
& =\frac{T_{1}}{T_{0}-T_{1}} \frac{J_{0}+\frac{U_{3}}{U_{1}} x^{2} J_{2}+x^{4}\left(\frac{U_{5}}{U_{1}} J_{41}+\frac{U_{3}^{2}}{U_{1}^{2}} J_{42}\right)+\cdots+}{U_{3}} x^{2} \theta_{2}^{\prime}(0)+x^{4}\left(\frac{U_{5}}{U_{1}} \theta_{41}^{\prime}(0)+\frac{U_{3}^{2}}{U_{1}^{2}} \theta_{42}^{\prime}(0)\right)+\cdots+
\end{aligned}
$$

where the prime denotes differentiation with respect to $\eta$. Substitution of the values of $\theta_{0}^{\prime}(0), \theta_{2}^{\prime}(0), \theta_{41}^{\prime}(0), \theta_{42}^{\prime}(0)$ given by Newman (14) and of the values of $J_{0}, J_{2}, J_{41}, J_{42}$ given by Homsy, Geyling, and Walker (12) thus enables us to test the conjecture in Section 3 that the particle concentration, like the temperature, is constant over the cylinder surface, that is, that $C_{1}$ as given by [5.6] is independent of $x$, not only in the region near the stagnation point where $x$ is small but more generally.
We show in Table II the values of the parameters in the numerator of [5.6] given by Homsy, Geyling, and Walker for $\sigma=0.71$ and those in the denominator obtained by interpolation between the values for $\sigma=0.70$ and 1.00 given by Newman. The values of $C_{1} / C_{0}$ at $x=0$ obtained from the values of $J_{0}$ and $\theta_{0}^{\prime}(0)$ given by these authors and shown in column 3 of Table II recover those given for the case of flow past a cylinder in 
TABLE II

Parameters in the Expression [5.6] for the Particle Concentration at the Surface of a Cylinder in a Steady Stream of Fluid as a Function of Distance from the Forward Stagnation Point

\begin{tabular}{lllcccccc}
\hline & \multicolumn{1}{c}{$\frac{T_{1}}{T_{0}}$} & $\frac{T_{1} J_{0}}{\left(T_{0}-T_{1}\right) \theta_{0}^{\prime}(0)}$ & $\frac{J_{2}}{J_{0}}$ & $\frac{J_{41}}{J_{0}}$ & $\frac{J_{42}}{J_{0}}$ & $\frac{\theta_{2}^{\prime}(0)}{\theta_{0}^{\prime}(0)}$ & $\frac{\theta_{41}^{\prime}(0)}{\theta_{0}^{\prime}(0)}$ & $\frac{\theta_{42}^{\prime}(0)}{\theta_{0}^{\prime}(0)}$ \\
\hline 0.568 & 0.3333 & 0.4075 & 0.9117 & 1.198 & -0.3925 & 0.9030 & 1.182 & -0.3850 \\
0.568 & 0.5 & 0.5835 & 0.9079 & 1.191 & -0.3883 & 0.9030 & 1.182 & -0.3850 \\
0.568 & 0.7143 & 0.7825 & 0.9045 & 1.185 & -0.3857 & 0.9030 & 1.182 & -0.3850 \\
0.568 & 0.7778 & 0.8359 & 0.9034 & 1.183 & -0.3846 & 0.9030 & 1.182 & -0.3850 \\
0.568 & 0.8182 & 0.8681 & 0.9033 & 1.183 & -0.3846 & 0.9030 & 1.182 & -0.3850 \\
0.142 & 0.5 & 0.7534 & 0.9108 & 1.194 & -0.3891 & 0.9030 & 1.182 & -0.3850 \\
0.284 & 0.5 & 0.6762 & 0.9104 & 1.195 & -0.3894 & 0.9030 & 1.182 & -0.3850 \\
0.426 & 0.5 & 0.6236 & 0.9093 & 1.193 & -0.3891 & 0.9030 & 1.182 & -0.3850 \\
0.568 & 0.5 & 0.5835 & 0.9079 & 1.191 & -0.3883 & 0.9030 & 1.182 & -0.3850 \\
0.710 & 0.5 & 0.5510 & 0.9061 & 1.188 & -0.3872 & 0.9030 & 1.182 & -0.3850 \\
\hline
\end{tabular}

Note. The values of the $J$ parameters are taken from Homsy, Geyling, and Walker (12) and of the $\theta$ parameters from Newman (14).

Table I, although for different combinations of $H$ and $T_{1} / T_{0}$. The entries for $J_{2} / J_{0}, J_{41} /$ $J_{0}$ and $J_{42} / J_{0}$ are all remarkably constant, the variation being only $0.9,1.3$, and $2.0 \%$ respectively despite the wide variation in the values of $H$ and $T_{1} / T_{0}$. Furthermore, the values of $J_{1} / J_{0}, J_{41} / J_{0}$ and $J_{42} / J_{0}$ coincide, within the same margins, with the values of $\theta_{2}^{\prime}(0) / \theta_{0}^{\prime}(0), \theta_{41}^{\prime}(0) / \theta_{0}^{\prime}(0)$, and $\theta_{42}^{\prime}(0) / \theta_{0}^{\prime}(0)$, respectively, the small variation of $J_{2} / J_{0}, J_{41} /$ $J_{0}$ and $J_{42} / J_{0}$ being consistent with an approach to the expected exact equality as either $H \rightarrow 1$ or $T_{1} / T_{0} \rightarrow 1$. We may conclude first that that variation of particle concentration over the forward face of a cylinder in a stream is quite small, of the order of $1 \%$, for any cross-sectional shape, and second that this approximately constant value of the particle concentration at the cylinder surface is that given by the exact solution valid near the stagnation point.

\section{FLOW IN A CIRCULAR TUBE WITH NONUNIFORM WALL TEMPERATURE}

In this case, which may be regarded as the prototype for the second of the two groups of problems mentioned in Section 2, fluid with uniform temperature $T_{0}$ and particle concentration $C_{0}$ enters a tube of circular cross section with radius $R$ and the steady velocity distribution

$$
u=\frac{U}{U_{0}}=1-\frac{r^{2}}{R^{2}}
$$

is established. The wall temperature $T_{\mathrm{w}}$ is constant and equal to $T_{0}$ as far as a cross section to be defined as $x=0$, and thereafter falls, with consequent flux of both heat and particles to the wall. One form of wall temperature change for which some analytical progress with the temperature distribution is clearly possible is a discontinuous jump to a lower wall temperature $T_{1}$ at $x=0$, with $T_{\mathrm{w}}$ $=T_{1}$ at $x>0$. The thermophoretic deposition of particles resulting from this form of wall temperature variation has been considered in a paper by Walker, Homsy, and Geyling (7). These authors present some useful numerical data for the particle concentration which we shall extend and interpret with the aid of the general considerations given in the preceding sections. Several different aspects of the problem will be discussed separately. 


\section{The Temperature Boundary Layer at Short} Distances Downstream from a Jump in $T_{\mathrm{w}}$

At sufficiently small distances downstream from $x=0$, the lower wall temperature diffuses laterally into fluid in which the velocity is proportional to distance from the wall and the wall is effectively plane. This gives rise to a type of temperature boundary layer whose features are well known and in which the temperature is a function only of the similarity variable $y(\gamma / \alpha \mathrm{x})^{1 / 3}$, where $y$ is normal distance from the wall and $\gamma$ is the velocity gradient at the wall, here equal to $2 U_{0} / a$. The particle concentration clearly will also be a function only of this same similarity variable. Walker, Homsy, and Geyling give integral expressions for the temperature $T$ and concentration $C$, and evaluate numerically $C_{1}$ (the uniform value of $C$ at the wall) for several different values of $T_{1} / T_{0}$ and $H$.

This is thus another exact similarity solution for $C$ which can be used as a test of the general propositions put forward in Section 3 for all cases in which the particle trajectories begin in a region where the temperature and concentration have uniform values of $T_{0}$ and $C_{0}$. However, no detailed consideration of the numerical values of $C_{1} / C_{0}$ as a function of $T_{1} / T_{0}$ and of $H$ is needed because, as may be seen from Table I, they are close to those for the three flow fields examined in Section 4 and especially close to those for the case of boundary-layer flow over a semi-infinite flat plate which is cooler than the oncoming stream. The conclusions reached in Section 4 concerning these latter flow fields thus apply equally to this new similarity flow field.

\section{Nonsmall Distances Downstream from a Wall Temperature Jump}

When the thickness of the temperature boundary layer is no longer small compared with the tube radius (that is, when the condition $\left(x \alpha R / U_{0}\right)^{1 / 3} \ll R$ is no longer satisfied), the above similarity solution is not applicable.
Walker, Homsy, and Geyling obtained the distributions of temperature and concentration at these larger values of $x$ numerically. $T$ was found from a numerical solution of Eq. [2.8] (with $\partial T / \partial t=0$, and $\alpha=$ const., and temperature gradients in the $x$-direction being neglected on the assumption that the Péclet number is large), and then $C$ was found by integrating the other governng equation [2.9] along a particle trajectory. Their results for the rate of deposition of particles on the tube wall are expressed in terms of the "cumulative efficiency" $E(x)$, defined as the fraction of the particles carried past the section $x=0$ that is deposited on the tube wall between $x=0$ and the section $x$, that is,

$$
\begin{aligned}
E(x) & =\frac{\int_{0}^{x} J(x) 2 \pi R d x}{\frac{1}{2} \pi R^{2} U_{0} C_{0}} \\
& =\frac{4 H\left(T_{0}-T_{1}\right)}{R U_{0} T_{1}} \int_{0}^{x} \frac{C_{\mathrm{w}}}{C_{0}} \frac{F(x)}{T_{0}-T_{1}} d x,
\end{aligned}
$$

where $F$ is the local heat flux to the wall divided by $\rho c_{\mathrm{p}}$, as in [2.11], and $F /\left(T_{0}-T_{1}\right)$ is independent of $T_{0} / T_{1}$. The heat flux $F$ diminishes to zero as the temperature in the fluid stream relaxes to that of the wall, and $E(x)$ asymptotes to the value $E_{\infty}$ as $x \rightarrow \infty$. Walker, Homsy, and Geyling give graphs of $E(x)$ as a function of $x$ for a few different values of $H$ and of the temperature ratio $T_{1} / T_{0}$ (viz., $H=1, T_{1} / T_{0}=\frac{1}{3}$ and $\frac{2}{3} ; H$ $\left.=0.7, T_{1} / T_{0}=\frac{1}{2}\right)$ and a table of the values of $E_{\infty}$ for many more combinations of values of $H$ and $T_{1} / T_{0}$.

If this problem is now approached with the help of the general considerations of Section 3, and if in particular we adopt the hypothesis that $C$ is constant over any surface on which $T$ is constant, then $C_{\mathrm{w}}$ has the constant value $C_{1}$ on the tube wall for $x>0$ and the expression [6.3] for the cumulative efficiency becomes 


$$
E(x)=\frac{4 H C_{1}\left(T_{0}-T_{1}\right)}{R U_{0} C_{0} T_{1}} \int_{0}^{x} \frac{F}{T_{0}-T_{1}} d x
$$

showing a proportionality of the amounts of particles and of heat transferred to the wall over the axial distance $x$. Unfortunately Walker, Homsy, and Geyling do not record their calculated values of $C_{\mathrm{w}}$ as a function of $x$, so we are not in a position to make a direct check of the hypothesis that $C_{\mathrm{w}}$ is constant (for $x>0$ ). However, we can make several indirect checks.

As we know, there is an exact solution [3.2] when $H=1$, and in that case [6.3] becomes, without approximation,

$$
\begin{aligned}
\{E(x)\}_{H=1} & \\
& =\frac{4\left(T_{0}-T_{1}\right)}{R U_{0} T_{0}} \int_{0}^{x} \frac{F}{T_{0}-T_{1}} d x .
\end{aligned}
$$

The approximate relation [6.4] is thus equivalent to

$$
\begin{gathered}
\frac{C_{0} T_{1}}{H C_{1}\left(T_{0}-T_{1}\right)} E(x)=\frac{T_{0}}{T_{0}-T_{1}}\{E(x)\}_{H=1} \\
=\frac{4}{R U_{0}} \int_{0}^{x} \frac{F}{T_{0}-T_{1}} d x,
\end{gathered}
$$

indicating that the quantity on the far left is the same function of $x$ for all values of $H$ and $T_{1} / T_{0}$. This is exactly what Walker, Homsy, and Geyling noticed for three sets of calculations for different combinations of values of $H$ and $T_{1} / T_{0}$ (see their Fig. 2).

For the asymptotic value of $E$ we may use the fact that the total transfer of heat to the wall is equal to the difference between the amounts of heat carried by the fluid past the sections $x=0$ and $x \rightarrow \infty$, where the fluid temperatures are $T_{0}$ and $T_{1}$, respectively. This corresponds to

$$
\int_{0}^{\infty} F(x) 2 \pi R d x=\frac{1}{2} \pi R^{2} U_{0}\left(T_{0}-T_{1}\right),
$$

whence we find first the exact result

$$
\left(E_{\infty}\right)_{H=1}=\frac{T_{0}-T_{1}}{T_{0}}
$$

from [6.5] and second, from [6.4], the approximate result

$$
E_{\infty}=H \frac{T_{0}-T_{1}}{T_{1}} \frac{C_{1}}{C_{0}} .
$$

Table III shows the values of $E_{\infty}$ calculated (by numerical integration) for a number of pairs of values of $H$ and $T_{1} / T_{0}$ by Walker, Homsy, and Geyling (7) and for comparison the values of $E_{\infty}$ which are obtained from [6.9] when the simple expression [3.8] for the particle concentration is used, viz.,

$$
\begin{aligned}
E_{\infty}=H & \frac{T_{0}-T_{1}}{T_{0}} \\
& \times\left\{1+(1-H) \frac{T_{0}-T_{1}}{T_{0}}\right\} .
\end{aligned}
$$

\section{TABLE III}

\begin{tabular}{llllll}
\multicolumn{6}{c}{ Values of $E_{\infty}$} \\
\hline & \multicolumn{5}{c}{$T_{1} / T_{0}$} \\
\cline { 2 - 6 } 1.0 & 0.2 & 0.3 & 0.5 & 0.6 & 0.8 \\
\hline \multirow{2}{*}{0.8} & 0.800 & 0.667 & 0.500 & 0.333 & 0.200 \\
& 0.800 & 0.667 & 0.500 & 0.333 & 0.200 \\
& 0.73 & 0.59 & 0.42 & 0.27 & 0.16 \\
0.7 & 0.68 & 0.54 & 0.39 & 0.24 & 0.14 \\
& 0.694 & 0.560 & 0.403 & 0.257 & 0.148 \\
0.5 & 0.58 & 0.44 & 0.30 & 0.18 & 0.10 \\
& 0.560 & 0.444 & 0.313 & 0.194 & 0.110 \\
0.3 & 0.42 & 0.31 & 0.20 & 0.12 & 0.06 \\
& 0.374 & 0.293 & 0.203 & 0.123 & 0.068 \\
\hline
\end{tabular}

Note. Each of the two figures in an entry represents $E_{\alpha}$, the fraction of the number of particles entering a circular tube that is deposited on the wall downstream of the cross section where there is a sudden drop of the wall temperature from $T_{0}$ to $T_{1}$. The upper of the two figures is the value found by numerical integration of the governing equations by Walker, Homsy, and Geyling (7), and the lower is the value which follows when $C$ and $T$ are assumed to be related as in [3.8]. 
The agreement is quite good, except when both $H$ and $T_{1} / T_{0}$ are small.

If we are willing to adopt the approximate relation between $C / C_{0}$ and $T / T_{0}$ suggested in [3.8], then [6.6] can be written as

$$
\begin{aligned}
E(x)=H\{1+(1-H) & \left.\frac{T_{0}-T_{1}}{T_{0}}\right\} \\
& \times\{E(x)\}_{H=1}
\end{aligned}
$$

which in conjunction with Walker, Homsy, and Geyling's calculations of $\{E(x)\}_{H=1}$ provides explicit values of $E(x)$ for any values of $H$ and $T_{1} / T_{0}$. (They give values of $E$ as a function of $x$ for $H=1, T_{1} / T_{0}=\frac{1}{2}$ in their table II-and a plot of this function in their Fig. 3-and the corresponding values of $\{E(x)\}_{H=1}$ for other values of $T_{1}-T_{0}$ follow from the exact relation $\{E(x)\}_{H=1} \propto\left(T_{0}\right.$ $\left.-T_{1}\right) / T_{0}$.) Alternatively it may be more convenient for computational purposes to use the series formula for the heat flux to the tube wall found originally by Graetz (15), viz.,

$$
\begin{aligned}
& \frac{F}{T_{0}-T_{1}} \\
& \left.\quad=\frac{\alpha}{\mathrm{R}} \sum_{n=0}^{\infty} c_{n} \psi_{n}^{\prime}(1) \exp \left(-\beta_{n}^{2} x / R\right]^{\Phi}\right),
\end{aligned}
$$

where $\Phi=R U_{0} / \alpha$ and $c_{n}, \psi_{n}^{\prime}(1)$, and $\beta_{n}$ are numbers whose values may conveniently be obtained from the paper by Sellars, Tribus, and Klein (16). The substitution of [6.12] in [6.5] gives

$$
\begin{aligned}
\{E(x)\}_{H=1}= & \frac{4\left(T_{0}-T_{1}\right)}{T_{0}} \sum_{n=0}^{\infty} \frac{c_{n} \psi_{n}^{\prime}(1)}{\beta_{n}^{2}} \\
& \times\left\{1-\exp \left(-\beta_{n}^{2} x / R[0)\right\} .\right.
\end{aligned}
$$

We have evaluated $\{E(x)\}_{H=1}$ from the formula [6.13] and find close agreement with the values obtained by Walker, Homsy, and Geyling (7) from a numerical integration of the equation for the temperature in the fluid.

\section{Other Forms of Transition from One} Uniform Wall Temperature to Another

Suppose now that the wall temperature is $T_{0}$ at $x \leqslant 0$ and $T_{1}$ at $x \geqslant x_{1}$, and that $T_{\mathrm{w}}$ decreases monotonically from the value $T_{0}$ at $x=0$ to the value $T_{1}$ at $x=x_{1}$. The cumulative efficiency is then given by

$$
\begin{aligned}
E(x) & =\frac{\int_{0}^{x} J(x) 2 \pi R d x}{\frac{1}{2} \pi R_{2} U_{0} C_{0}} \\
& =\frac{4 H}{R U_{0} T_{0}} \int_{0}^{x} \frac{C_{\mathrm{w}}}{C_{0}} \frac{T_{0}}{T_{\mathrm{w}}} F(x) d x .
\end{aligned}
$$

Again some useful exact results can be derived for the case $H=1$. With the aid of [3.2] we find

$$
\{E(x)\}_{H=1}=\frac{4}{R U_{0} T_{0}} \int_{0}^{x} F(x) d x,
$$

and then, from the heat-conservation relation [6.7],

$$
\left(E_{\infty}\right)_{H=1}=\frac{T_{0}-T_{1}}{T_{0}} .
$$

Thus when $H=1$ the asymptotic cumulative efficiency is independent of the way in which the temperature changes between the values in the two uniform regions far upstream and far downstream.

Walker, Homsy, and Geyling (7) calculated the cumulative efficiency from a numerical integration of the governing equations for a linear variation of the wall temperature between $x=0$ and $x=0.3 R \mathrm{P}$ in the one case $T_{1} / T_{0}=\frac{1}{2}, H=1$, and noted empirically that the asymptotic value of $E$ had the same value $\left(\frac{1}{2}\right)$ as in the case of a jump in wall temperature at $x=0$ with $T_{1} / T_{0}=\frac{1}{2}$.

\section{DISCUSSION}

In this paper we have taken a rather pragmatic view of the problem of estimating rates of thermophoretic deposition of small particles in flow over cold rigid surfaces.

We have supposed the availability of an 
expression like [2.1] with [2.2] for the speed with which a spherical particle of given size and thermal conductivity moves down an ambient temperature gradient in consequence of the discrete molecular structure of the gas. With that assumption, and provided the fluid velocity distribution is known, the problem reduces to the finding of solutions of the two coupled differential equations for the temperature and particle concentration in the fluid. We have considered a number of specific flow fields for which results concerning the temperature and particle concentration distributions are already available in the literature, and after extending and comparing these results we have used them as tests of the value and accuracy of the general hypothesis and approximations described in Section 3.

It appears that, in cases in which all the particles originate in an upstream region in which the fluid temperature and particle concentration are uniform, the concentration at a point in the fluid is nearly determined fully by the local temperature (for a given value of $H$ ), with the consequence that the particle concentration is approximately uniform at a cold boundary whose temperature is uniform. This approximate uniformity is exact in the four different flow fields described herein in which the concentration and temperature are functions of a single "similarity" position variable (such as $y / x^{1 / 2}$ ) and it is exact also for all flow fields when $H=1$; and in two "nonsimilarity" cases (flow in the boundary layer on much of the forward face of a cold cylinder in a stream, and flow in a circular tube at nonsmall distances downstream from a sudden temperature drop) the available numerical data indicate uniformity of the concentration at the cold isothermal boundary to a good approximation. The value of this conclusion lies in the fact that when $C$ and $T$ are both uniform over a cold boundary (with values $C_{1}$ and $T_{1}$ ) the total particle flux by thermophoresis to that boundary becomes

$$
\int J d A=\frac{H C_{1}}{T_{1}} \int F d A
$$

showing a proportionality to the total heat flux to the boundary which is a more readily measured quantity.

Furthermore, the relation between the values of $C$ and $T$ at the cold isothermal boundary is approximately the same for all the specific flow fields considered here. Table I shows the closeness of the values of $C_{1} / C_{0}$ for the four boundary-layer similarity cases, and the closeness in the two nonsimilarity cases is implicit in Tables II and III. This raises the possibility of devising an approximate universal relation between $C_{1} / C_{0}$ and $T_{1} / T_{0}$ which conforms to the analytical constraints listed in Section 3 and which fits the available numerical data well. One such relation which has the merit of extreme simplicity is that indicated in [3.8], viz.,

$$
\frac{C_{1}}{C_{0}}=\frac{T_{1}}{T_{0}}\left\{1+(1-H)\left(\frac{T_{0}-T_{1}}{T_{0}}\right)\right\}
$$

The comparison between the values of $C_{1} /$ $C_{0}$ given by this relation and those obtained by numerical integration in the four boundary-layer similarity cases made in Table I shows differences of at most a few percent over a wide range of values of the parameters $H$ and $T_{1} / T_{0}$. The use of the relation [7.2] also gives accurate results for the flux of particles to the boundary in the case of a boundary layer over a substantial part of the forward face of a cylinder in a stream, and in the case of flow in a tube with a sudden drop in the wall temperature except when $H$ and $T_{1} / T_{0}$ are both small (see Table III). Functions of $T_{1} / T_{0}$ and $H$ which fit the calculated values of $C_{1} / C_{0}$ even more accurately than [7.2] can be invented easily.

The conclusion is that the expression [7.1] for the total particle flux to an isothermal cold boundary, with the subsidiary relation 
[7.2], reproduces all the available numerical results, viz., those for the four similarity temperature fields and the two nonsimilarity temperature fields, with an accuracy which would be sufficient in practical applications.

We suggest that there is an adequate basis for the use of [7.1] and [7.2], with due caution, for the prediction of the rates of thermophoretic deposition of small particles in the case of flow systems in which the velocity distribution is either unknown or of too complex a form for the numerical calculation of the distributions of temperature and particle concentration to be feasible. It should be kept in mind, however, that a condition for the applicability of [7.1] and [7.2] is that all the particles that are deposited on a cold isothermal boundary at temperature $T_{1}$ originate in an upstream region where the temperature and particle concentration are uniform with values $T_{0}$ and $C_{0}$, respectively.

It may also be noted that the arguments used in Section 4 do not require the flow to be steady. The flow could be turbulent, for instance. The assertion that $(\partial / \partial \tau+\mathbf{u} \cdot \nabla) T$ is zero or negative everywhere in the fluid cannot then be made with confidence, but it remains true that the ensemble average of this material derivative is negative and this seems likely to be sufficient for the validity of the bounds [3.6].

A case of considerable practical interest for which direct computation of deposition rates is unlikely to be feasible and for which [7.1] and [7.2] may therefore be useful is a cold circular cylinder in a stream of fluid carrying small particles, with the Reynolds number of the flow about the cylinder much larger than unity. (The circular cylinder might be a rod in a heat exchanger or a thermal precipitator.) There exist formulae for the total heat transfer rate which are known to fit the experimental data over particular ranges of the Reynolds number (see for instance Incropera and De Witt (17)), and these in conjunction with [7.1] and [7.2] could be used for the prediction of the total particle transfer rate. If it were possible, observation of the total particle transfer rate in this case of a circular cylinder in a stream would be very valuable because that would provide a test of the formulae [7.1] and [7.2] on a flow system which is much more complex than those considered in this paper. The value of $H$ for a particle of given size and thermal conductivity cannot be said to be known reliably, but one could get around that difficulty by observation of the total particle transfer rate at more than one temperature ratio or more than one Reynolds number.

\section{REFERENCES}

1. Kennard, E. H., "Kinetic Theory of Gases," McGraw-Hill, New York, 1938.

2. Hidy, G. M., and Brock, J. R., "The Dynamics of Aerocolloidal Systems," Pergamon, Elmsford, N.Y., 1970.

3. Friedlander, S. K., "Smoke, Dust and Haze," WileyInterscience, New York, 1977.

4. Talbot, L., Cheng, R. K., Schefer, R. W., and Willis, D. R., J. Fluid Mech. 101, 737 (1980).

5. Shen, C. Mech. Sinica 1, 7 (1983).

6. Derjaguin, B. V., Rabinovich, Ya. I., Storozhilova, A. I., and Shcherbina, G. I., J. Colloid Interface Sci. 57, 45 (1976).

7. Walker, K. L., Homsy, G. M., and Geyling, F. T., J. Colloid Interface Sci. 69, 138 (1979).

8. Goren, S. L., J. Colloid Interface Sci. 61, 77 (1977).

9. Weinberg, M. C., J. Colloid Interface Sci. 84, 550 (1981).

10. Goldstein, S. (Ed.), "Modern Developments in Fluid Mechanics." Oxford Univ. Press (Clarendon), London, 1938.

11. Sibulkin, M., J. Aerosp. Sci. 19, 570 (1952).

12. Homsy, G. M., Geyling, H. T., and Walker, K. L., J. Colloid Interface Sci. 83, 495 (1981).

13. Schlichting, H., "Boundary Layer Theory," 4th ed., McGraw-Hill, 1960.

14. Newman, J., Int. J. Heat Mass Transfer 9, 705 (1966).

15. Graetz, L., Ann. Phys. Chem. 25, 337 (1885).

16. Sellars, J. R., Tribus, M., and Klein, J. S., Trans. ASME 78, 441 (1.956)

17. Incropera, F. P., and De Witt, D. P., "Fundamentals of Heat Transfer." Wiley, New York, 1981. 